\title{
Improving planning and post-operative assessment for Total Hip Arthroplasty
}

\author{
Benedikt Magnússon (1,2), Pröstur Pétursson (3), Kyle Edmunds (1), Gígja Magnúsdóttir (4), \\ Grétar Halldórsson (4), Halldór Jónsson jr. $(5,6)$, Paolo Gargiulo $(1,7)$ \\ (1) Institute for Biomedical and Neural Engineering, Háskólinn í Reykjavík, Reykjavík, \\ Iceland; (2) Department of Clinical Engineering, Landspítali Hospital, Reykjavík, Iceland; (3) \\ Department of Systems and Solutions, Landspitali Hospital, Reykjavík, Iceland (4) \\ Rehabilitation Clinic Grensás Landspítali Hospital, Reykjavík, Iceland; (5) Orthopedic Clinic, \\ Landspítali Hospital, Reykjavík, Iceland; (6) Medical Faculty, University of Iceland, \\ Reykjavík, Iceland; (7) Department of Science, Landspítali University Hospital, Iceland
}

\begin{abstract}
Total Hip Arthroplasty (THA) remains the gold standard of treatment for patients who suffer with a variety of hip-related pathological degeneration or trauma. These patients often exhibit significantly less post-operative pain and an increase in the range of motion of the joint, but there are still relatively common instances of debilitating periprosthetic complications that call into question the method for pre-surgical implant choice. Currently, there are two principal options for THA prostheses: cemented or non cemented. Utilizing the cemented procedure ensures a faster acquisition of adequate implant stability than with the non cemented procedure, but can eventually lead to an increased periprosthetic fracture risk. Non cemented prosthetic stems are more frequently revised within the first few years following THA due to periprosthetic fracture, but non cemented revision surgeries generally result in fewer complications than those of cemented implants. Surgeons typically rely on experience or simple patient metrics such as age and sex to prescribe which implant procedure is optimal, and while this may work for most patients, there is a clear need to analyze more rigoriously patient conditions that correlate to optimal post-THA outcomes. The results from the investigation reported herein indicate that an understanding of how the percent composition and quality of a patient's quadriceps muscle in both healthy and operated legs may be a better indicator for prosthetic choice. Additionally, these data emphasize that the traditional metrics of age and sex inadequately predict changes in quadriceps composition and quality and thereby have comparatively minor utility in determining the patient-appropriate prosthetic type.

Key Words: Total Hip Arthroplasty, Prosthetic selection, Muscle size and quality, Anatomical modeling, Surgical planning.
\end{abstract}

For decades, Total Hip Arthroplasty (THA) has been the gold standard of treatment for patients suffering with osteoarthritis of the hip or fracture-induced osteonecrosis of the femoral head or neck. While this procedure remains one of the most efficacious modalities for both reducing patient pain and restoring hip function, post-operative complications and revisions are relatively common, primarily due to localized fracture or periprosthetic unloading. ${ }^{1,2}$ The procedure has historically been prevalent in countries with relatively high life spans, like the Nordic community. ${ }^{3-5}$ However, despite the prevalence of the procedure in these countries, annual reports from the national arthroplasty registries indicate differences in choices of implant brands, fixation methods, and overall prosthetic survival - a notion which necessitates the development of an innovative and collaborative approach to THA planning. ${ }^{6-8}$

Currently, there are two principal options for THA prostheses: cemented or non cemented. Utilizing the cemented procedure ensures a faster acquisition of adequate implant stability than with the non cemented procedure, but can eventually lead to an increased periprosthetic fracture risk due to the reduction in local bone density from reduced local mechanical stress. ${ }^{9}$ Without the use of cement, prosthetic stability initially 


\section{Improving planning and assessment for THA}

Eur J Transl Myol - Basic Appl Myol 2015; 25 (2): 101-108

relies on frictional forces generated by geometrical interlocking (press-fitting), but is then secured by bone ingrowth into the femoral contact surface - a phenomenon encouraged by the preloaded fitting. ${ }^{10}$ Non cemented prosthetic stems are more frequently revised within the first few years following THA due to periprosthetic fracture, but non cemented revision surgeries generally result in fewer complications than those of cemented implants. ${ }^{11-15}$ Nonetheless, revision always presents a major challenge for orthopedic surgeons because of the poor quality of the surrounding bone. ${ }^{16}$

Current surgical philosophy dictates that a non cemented prosthesis should only be used if a patient's femur can withstand both the forces incurred by compressive stresses during the press-fitting and the functional loading after the operation. Additional consideration is given to whether eventual bone ingrowth can be both predictable and sufficient. Unfortunately, there is no straightforward approach to make this assessment, since quantitative pre-operative bone quality measurement is not performed, despite knowing that it would have a positive influence on the success of the operation. Additionally, the use of extant literature resources is problematic since large studies comparing non cemented to cemented THA have shown contrasting outcomes. ${ }^{17}$ Instead, orthopedic surgeons must carefully evaluate each particular patient's individual situation and choose an optimal protocol based upon acquired or shared intuition - a difficult task for novice surgeons. Initially, both the patient's age and gender are taken into account, since bone mineralization decreases with aging and differs between men and women. ${ }^{18,19}$ In general, cemented implants are more frequently used for older, less active people and/or people with weak bones, while non cemented implants are more frequently used for younger and/or more active people. However effective these generalizations may be in prescribing THA procedures, there may be many other relevant differences between individual patients; thus, the development of a patient-specific, quantitative methodology is needed.

After THA, patients' gaits typically exhibit improvement, but still exhibit asymmetries even after full recovery. ${ }^{20-24}$ However, even with the continued development of gait analysis techniques there is a general lack of understanding regarding joint motion of the lower extremities in the THA population, which can make certain patient's rehabilitation challenging and lengthy. The densities of the quadriceps muscles in patients undergoing THA have been shown to correlate with femoral bone mineral density. Both bone and muscle densities tend to be lower in the operated side which may be caused by the patient's shielding the involved side due to pain. $^{25}$ However, muscular strength tends to increase substantially on this side following the first post-operative year. ${ }^{26}$ The

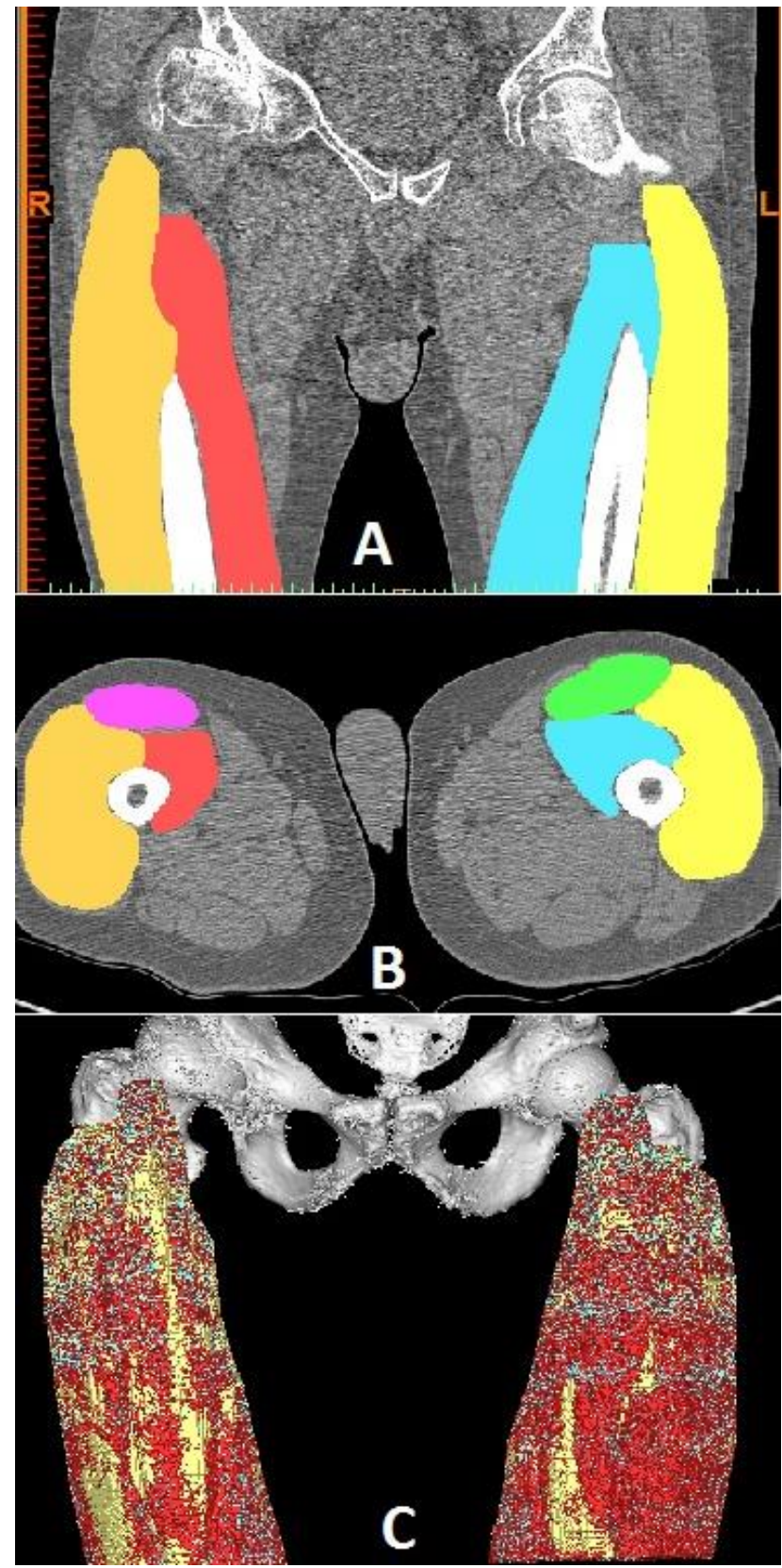

Fig 1. A) CT slice in a coronal view showing the quadriceps muscles that are individually segmented. B) Axial view showing the segmentation of the quadriceps muscles in vastus lateralis, rectus femoris and vastus medialis. C) The quadriceps muscles and the femur bone in a $3 D$ view after segmentation. The colors red, cyan and yellow represent respectively: muscle, dense connective and fat as defined in Gargiulo $P$, et al. ${ }^{27}$

functionality of the muscles is correlated with the muscle composition. Not only is the higher percentage of muscle fibers compared to fat generally linked to a more active muscle, but also the quality of the muscle fibers. $^{27}$ 
A

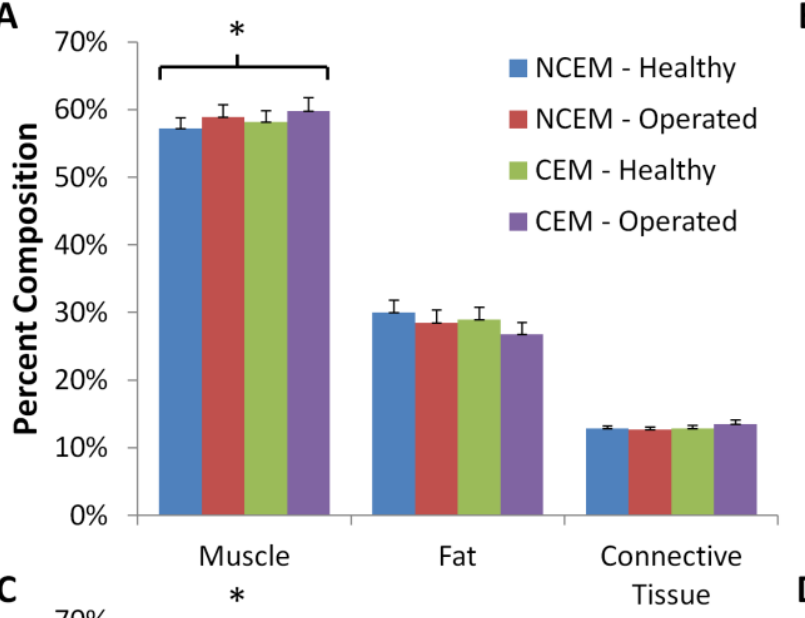

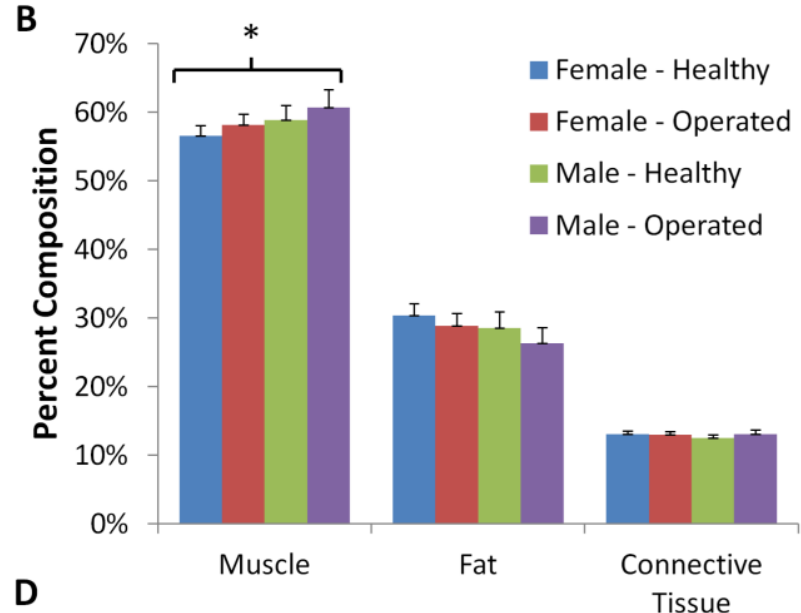

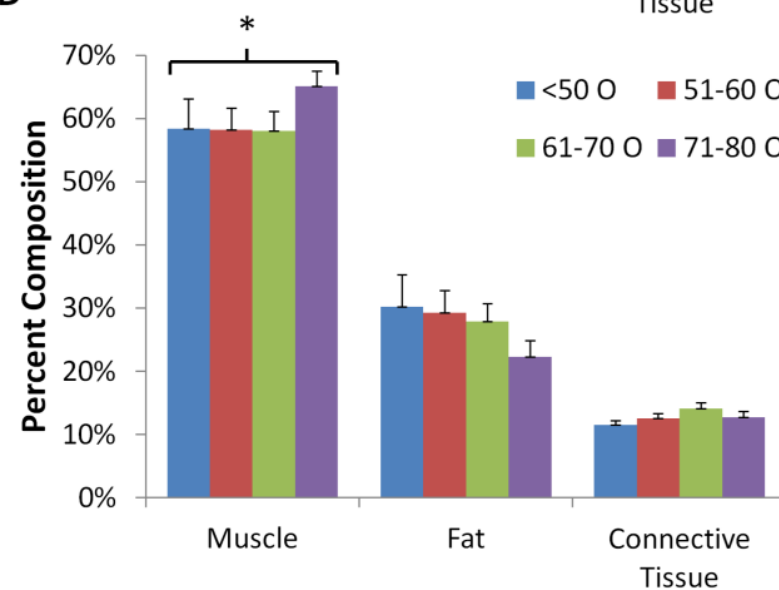

Fig 2 Shows the average composition of the quadriceps muscles for each of the specific patient groups and healthy versus operated legs. A) Compositions from patients with cemented (CEM) versus non cemented (NCEM) implants. B) Compositions from all female patients versus all males patients. $\boldsymbol{C}$ ) and $\boldsymbol{D})$ Compositions from healthy $(\boldsymbol{C})$ and operated $(\boldsymbol{D})$ legs, from patients within each of the four previously-mentioned age groups. Note that in each patient group, muscular percent composition was significantly higher than either fat or connective tissue percentages in all conditions $(*: p<0.05)$.

The research presented here was aimed at investigating the density distribution patterns and overall muscular quality of both operated and healthy quadriceps in patients undergoing THA. Results from this investigation can contribute to the clinical understanding of pre-operative decision-making in regards to prescribing THA prosthetic type. Furthermore, developing an understanding of changes in quadriceps muscle quality can serve as an indicator for post-operative patient outcome, thereby helping to guide rehabilitation strategies via a patient-specific understanding of pre-operative muscular quality and density. This study is the first part of the post-op patients' muscle recovery assessment (the second part being the same measurements one year post-op). After one year it will be possible to correlate the quality of the muscles and the functionality of the muscles using these measurements along with gait measurements.

\section{Material and Methods}

\section{CT data acquisition}

EMG This study includes data from 68 patients: 38 females (an average age 64 years) and 30 males (an average age of 56 years) who were scheduled to undergo their first total hip arthroplasty. Of the 68 patients, 40 of them received a non cemented implant, while 28 received a cemented implant. The decision regarding prosthetic suitability was in the hands of the operating surgeon of each patient, who based his implant prescription on typical general patient characteristics or conditions, such as age and sex. All patients were scanned in a 64 slice Phillips Brilliance CT scanner. The scanning area reached from the iliac crest to the middle of the femur bone (Fig. 1).

CT dataset processing

After the scanning, all datasets were processed in MIMICS. The processing protocol was as follows. 


\section{Improving planning and assessment for THA}

Eur J Transl Myol - Basic Appl Myol 2015; 25 (2): 101-108
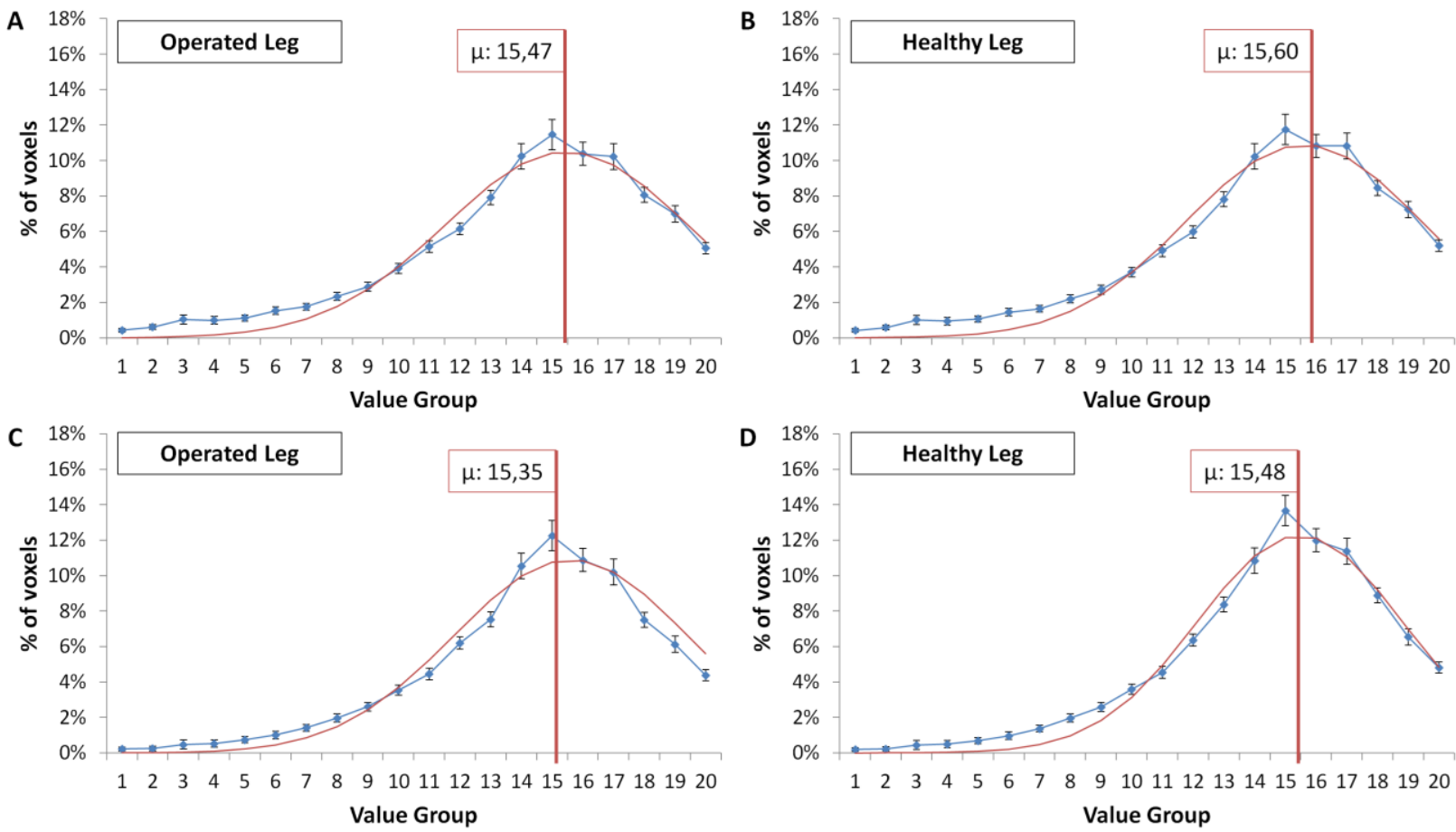

Fig 3. Hounsfield unit (HU) distribution from -135 to 200, divided into 20 bins. Each of the plots show mean values of each patient condition with computed standard error (blue curve). The plots on the left side show the operated leg histograms, while the plots on the right show the healthy leg. The red curve indicated the Gaussian curve fit for each HU distribution, and the vertical red line denotes the Gaussian centroid value, $" \mu " . \boldsymbol{A})$ and $\boldsymbol{B})$ are non cemented patients, while $\boldsymbol{C})$ and $\boldsymbol{D})$ are cemented patients.

Firstly, each of the quadriceps muscles of both legs were segmented. Secondly, the muscles were divided into sections according to tissue types (Fig. 1,C). ${ }^{27}$ Finally, all Hounsfield values were exported so they could be further analysed.

\section{Modeling of Hounsfield units distribution}

To discern the average Hounsfield values for each compared condition (prosthetic type, sex, and age), each CT scan was binned into 20 Hounsfield unit bins, generating a histogram of hounsfield unit values. These values were normalized to obtain histogram percentages, generating a normal distribution. This normal distribution was fitted to a theoretical Gaussian curve using the Solver function of Excel (Microsoft: 2007) and a generalized reduced gradient algorithm to maximize each $\mathrm{R} 2$ value and obtain an optimum curve fit. These distribution averages were then exported for further analysis.

\section{Statistical Analysis}

Statistical analyses were performed using appropriately sized ANOVA with posthoc testing carried out using a student's T-test. Differences were considered statistically significant for $\mathrm{p}<0.05$.

\section{Results}

Following segmentation and thresholding for muscular, fat, and connective tissues, it was possible to observe changes in tissue composition in both legs as a function of various patient parameters. Patients were sorted firstly according to the type of prosthesis selected by their surgeon, or cemented versus non cemented ( $\mathrm{n}$ of 28 versus 40, respectively). Secondly, patients were sorted according to their sex (n of 38 for females and 30 for males). Lastly, sorting was performed according to patient age, where patients were divided into four age groups: younger than 50 (n. of 10), between 51 and 60 (n. of 21), between 61 and 70 (n. of 26), and between 71 and 80 (n. of 11). The results from these analyses are shown in Figure 2.

Following the compositional analyses, each patient group's average hounsfield unit (HU) histogram was analyzed to discern both variation within patient groups and histogram location shift. To do this, each patient's CT scan was binned into 20 groups of $\mathrm{HU}$ values, ranging from -200 to 135 , and normalized to generate a patient-specific, normally-distributed histogram. Each of these histograms were then averaged to obtain an overall average curve for each patient group, which was then fit to a Gaussian distribution. The results from these analyses are displayed in Figures 3-5 for each respective patient group. As is apparent from Figure 3, when comparing the cemented and non cemented implant groups, the healthy leg distribution was shifted towards higher $\mathrm{HU}$ values, indicating the presence of more muscle in the 

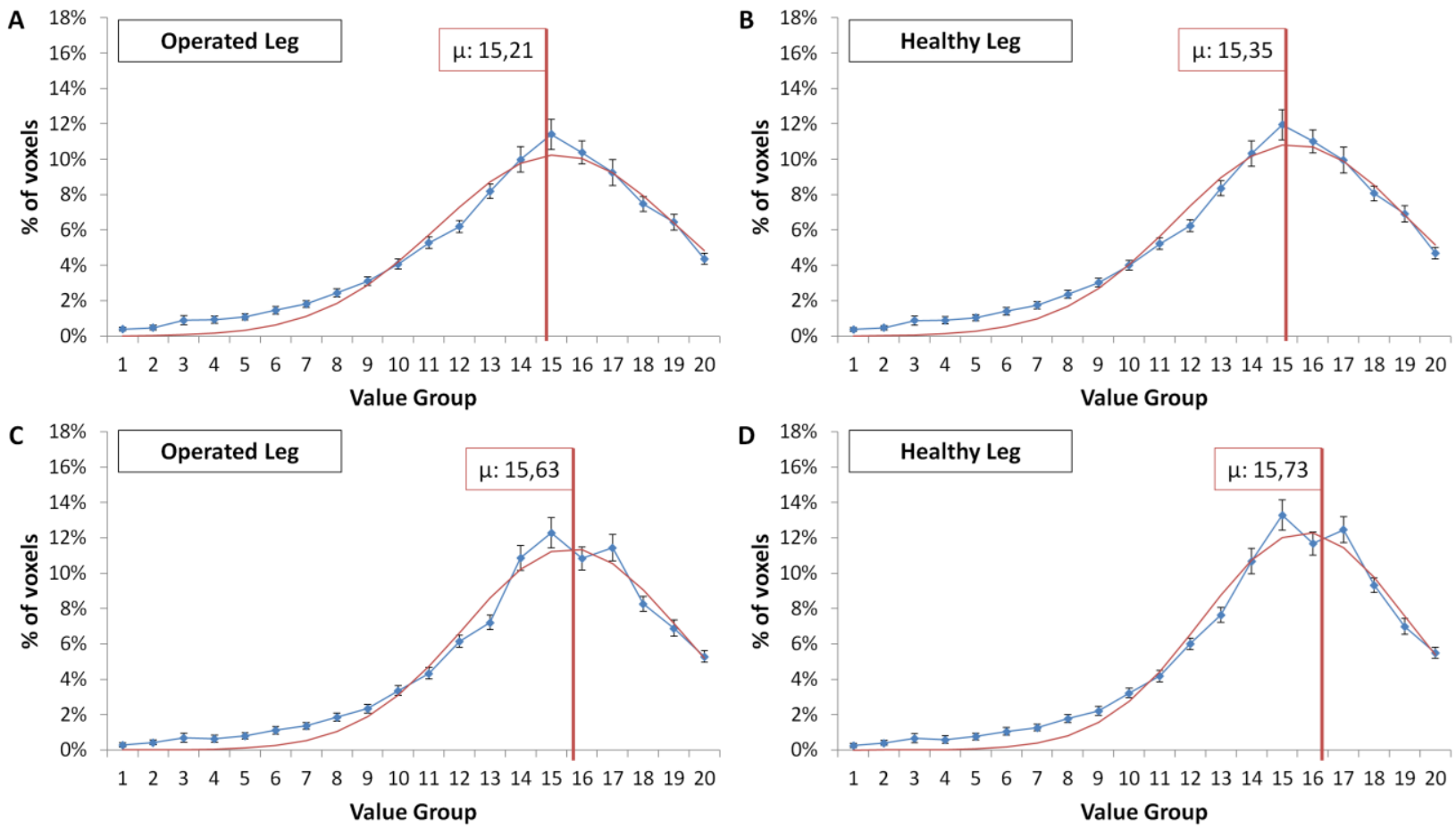

Fig 4. Binned hounsfield unit (HU) distribution for patients grouped by sex. The red and blue curves were generated analogously to those shown in Figure 3. $\boldsymbol{A}$ ) and $\boldsymbol{B}$ ) are female, while $\boldsymbol{C}$ ) and $\boldsymbol{D})$ are male patients.

healthy leg than within the operated leg. Additionally, HU values were higher in both legs in patients with the non cemented prosthesis compared to those who were given a cemented implant.

Figure 4: Binned hounsfield unit (HU) distribution for patients grouped by sex. The red and blue curves were generated analogously to those shown in Figure 3. A) and B) are female patients, while C) and D) are male patients.

When comparing female to male patients, what is immediately evident is that the HU peak is shifted to the right in both legs of male patients compared to those of females, indicating somewhat expectedly that male patients had higher muscular density in both legs. Additionally, once again patients of either sex exhibited higher $\mathrm{HU}$ centroids in healthy legs compared to those which were fitted with an implant, confirming the results depicted in Figure 3.

Finally, when comparing HU distributions for patients grouped according to age, youngest patients had much higher HU centroid values than those of older patients, with minimum values in both legs corresponding to the largest ( $\mathrm{n}$ of 16) patient group, or individuals from ages 61-70. Interestingly, once again in all conditions, average HU values were higher in the healthy leg than in the operated leg. In addition to the location of each HU curve peak, it is evident that patients in the oldest age group (71-80) exhibited much narrower HU distributions than those of other conditions, suggesting that within both legs of these patients, there was much less variation in the tissue type present.

\section{Discussion}

What is most evident from the data presented is that although the muscle composition of operated legs was higher than healthy legs in almost every patient group, the average HU distribution centroids were higher in healthy legs than in the operated side. A shift in average HU value has traditionally been an indicator of muscle quality, with higher $\mathrm{HU}$ values indicating a greater amount of muscle than fat or connective tissue. The presented results suggest that comparing percent composition to $\mathrm{HU}$ distribution does not necessarily correlate, but instead suggest that while muscular composition might be highest in the operated legs of patients, the overall muscular density, e.g. quality, may yet be lower in this leg compared to the healthy side. Further exploration into which analysis methodology best prescribes the capacity for the operated muscle to withstand both the implantation procedure and postsurgical rehabilitation is crucial to develop better decision-making in THA planning. In addition to this comparison of analysis methods, it also appears that males have higher percentages of muscle than females, but the differences between groups are not significantly different from one another. The same notion is true when comparing patient age and implant procedure. Alltogether the data suggest that there is no 


\section{Improving planning and assessment for THA}

Eur J Transl Myol - Basic Appl Myol 2015; 25 (2): 101-108
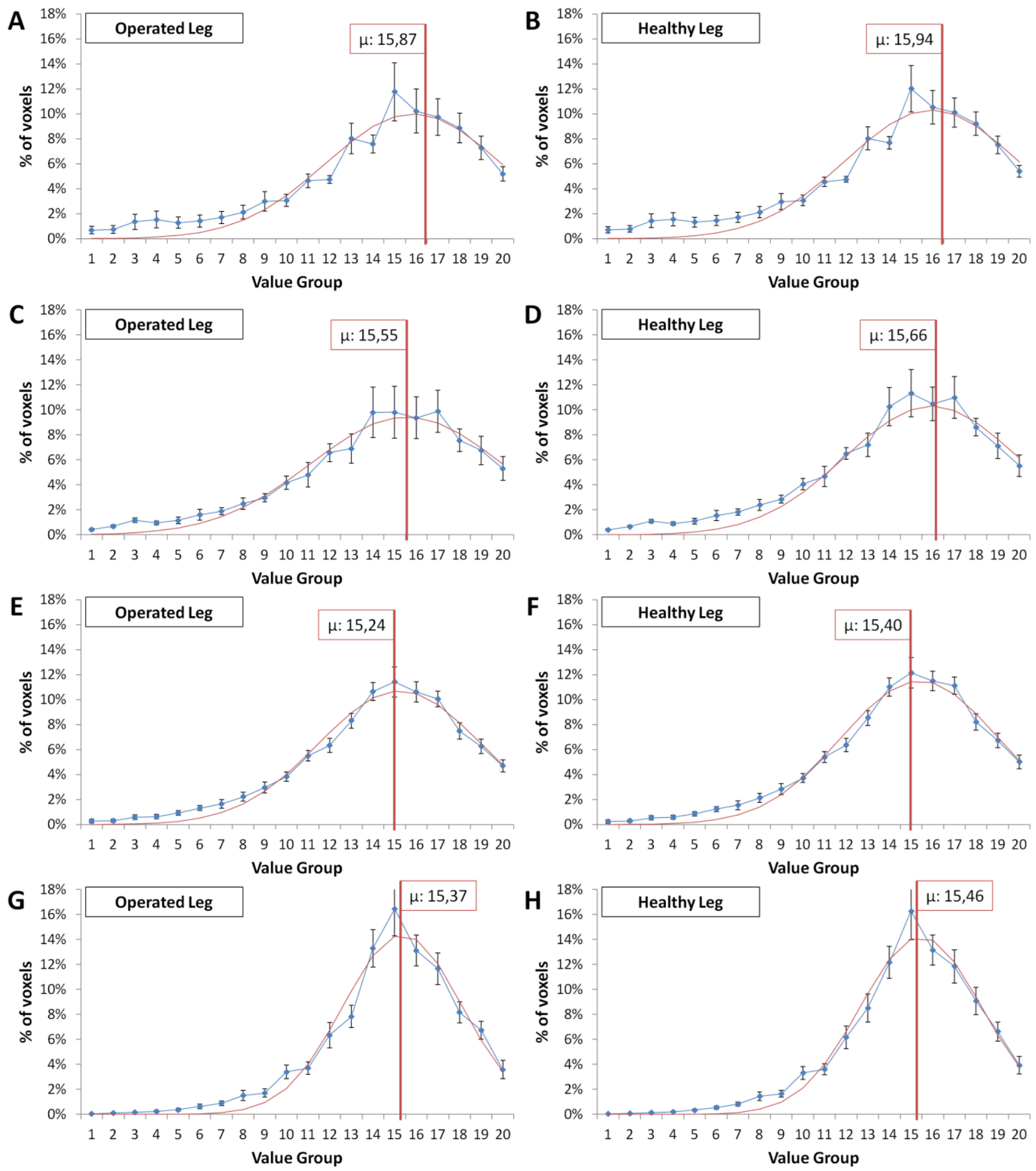

Fig 5. Binned hounsfield unit (HU) distribution for patients grouped by age. The red and blue curves were generated analogously to those shown in Figures 3 and 4 . A) and B) are patients less than 50 years old, $C)$ and D) are patients between 51 and 61 years old, E) and F) are patients between 61 and 70 years old, and $G$ ) and $H$ ) are patients between 71 and 80 years old.

clear utility in using traditional surgical metrics for governing THA prosthesis choice, such as age and sex. Finally, the distribution of $\mathrm{HU}$ values according to implant type indicates that the population of non cemented patients is more similar in distribution (smaller variance), which thereby confirms the doctors' 


\section{Improving planning and assessment for THA}

Eur J Transl Myol - Basic Appl Myol 2015; 25 (2): 101-108

decisions to give them non cemented implants. The cemented patients distribution show higher percentages of HU voxel values around those that correspond to muscular tissue, while these patients also exhibit larger variance in these values, indicating that some of the patients may have erroneously been prescribed a cemented implant, while a majority clearly received the optimal one.

All patients in this study are scheduled for one-year post-op visit where they will be scanned again. When the post-op data will be available, muscle recovery will be correlated with the different patient groups.

In conclusion, the comparison of muscle composition and $\mathrm{HU}$ distribution is a useful first step in developing a better understanding of the optimum THA procedure. Additionally, showing the similarities in muscle composition and quality between patients grouped by traditional planning metrics of age and sex suggests that surgical planning should incorporate additional patient conditions to identify the optimum THA planning strategy.

\section{Acknowledgement}

This research has been supported by the University Hospital Landspitali Scientific fund.

\section{Corresponding Author}

Benedikt Magnússon

Institute for Biomedical and Neural Engineering, Háskólinn í Reykjavík

Menntavegur 1

101 Reykjavík, Iceland

Office: 5435226, Mobile: 8245316

Email: benedikm@landspitali.is

E-mails of Co-Authors

Pröstur Pétursson, trosturp@landspitali.is

Kyle Edmunds, Kyle.Edmunds@Tufts.edu

Gígja Magnúsdóttir, gigjama@landspitali.is

Grétar Halldórsson, gretarh@landspitali.is

Halldór Jónsson jr., halldor@landspitali.is

Paolo Gargiulo, paologar@landspitali.is

\section{References}

1. van Loon CJ, de Waal Malefijt MC, Buma P, et al. Femoral bone loss in total knee arthroplasty. A review. ActaOrthop Belg 1999;65:154-63.

2. Radl R, Aigner C, Hungerford M, et al. Proximal femoral bone loss and increased rate of fracture with a proximally hydroxyapatite-coated femoral component. J Bone Joint Surg Br 2000;82:11515.

3. Mäkelä KT, Matilainen $\mathrm{M}$, Pulkkinen $\mathrm{P}$ et al. Countrywise results of total hip replacement. An analysis of 438,733 hips based on the Nordic Arthroplasty Register Association database. Acta Orthop 2014;85:107-16. doi: $10.3109 / 17453674.2014 .893498$.
4. Kurtz S, Ong K, Lau E, et al. Projections of primary and revision hip and knee arthroplasty in the United States from 2005. J Bone Joint Surg Am 2007;89:780-5.

5. Nemes S, Gordon M, Rogmark C, Rolfson O. Projections of total hip replacement in Sweden from 2013 to 2030. Acta Orthop 2014;85:238-43. doi: 10.3109/17453674.2014.913224. Epub 2014 Apr 23.

6. Havelin LI, Fenstad AM, Salomonsson R, et al. The Nordic Arthroplasty Register Association: A unique collaboration between 3 national hip arthroplasty registries with 280,201 THRs. Acta Orthop 2009;80:393-401. doi: $10.3109 / 17453670903039544$.

7. Wolf BR, Lu X, Li Y, et al. Adverse outcomes in hip arthroplasty: long-term trends. J Bone Joint Surg Am 2012;94:e103. doi: 10.2106/JBJS.K.00011.

8. Thien TM, Chatziagorou G, Garellick G, et al. Periprosthetic Femoral Fracture within Two Years After Total Hip Replacement: Analysis of 437,629 Operations in the Nordic Arthroplasty Register Association Database. J Bone Joint Surg Am 2014;96:e167. doi: 10.2106/JBJS.M.00643.

9. Bishop NE, Ferguson S, Tepic S. Porosity reduction in bone cement at the cement-stem interface. J Bone Joint Surg Br 1996;78:349-56.

10. Yamada H, Yoshiara Y, Henmi O, et al. Cementless total hip replacement: past, present and future. J Orth Sci 2009;14:228-41.

11. Engh CA, Glassman AH, Griffin WL, Mayer JG. Results of cementless revision for failed cemented total hip arthroplasty. Clin Orthop 1988;235:91-4.

12. Engelbrecht DJ, Weber FA, Sweet MB, Jakim I. Long-term results of revision total hip arthroplasty. J Bone Joint Surg 1990;72:41-7.

13. Lawrence JM, Engh CA, Macalino GE, Lauro GR. Outcome of revision hip arthroplasty done without cement. J Bone Joint Surg Am 1994;76:965-70.

14. Moreland JR, Bernstein ML. Femoral revision hip arthroplasty with uncemented, porous-coated stems. Clin Orthop 1995;319:141-6.

15. Hailer NP, Garellick G, Kärrholm J. Uncemented and cemented primary total hip arthroplasty in the Swedish Hip Arthroplasty Register. Acta Orthop 2010;81:34-41. 10.3109/17453671003685400.

16. Venesmaa PK, Kröger HP, Miettinen HJ et al. Monitoring of Periprosthetic BMD After Uncemented Total Hip Arthroplasty with DualEnergy X-Ray Absorptiometry, a 3-Year FollowUp Study. J Bone Min Res 2001;16:1056-61.

17. Radl R, Aigner C, Hungerford M, et al. Proximal femoral bone loss and increased rate of fracture with a proximally hydroxyapatite-coated femoral 


\section{Improving planning and assessment for THA}

Eur J Transl Myol - Basic Appl Myol 2015; 25 (2): 101-108

component. J Bone Joint Surg Br 2000; 82: 1151-5.

18. Magnússon B, Halldór Jónsson jr, Pröstur Pétursson, et al. Gait Analysis, bone and muscle density in THA patients, Basic Appl Myol 2012;22:155-60.

19. Taaffe DR, Cauley JA, Danielson M, et al. Race and sex effects on the association between muscle strength, soft tissue, and bone mineral density in healthy elders: the health, aging, and body composition study. J Bone Miner Res 2001;16: 1343-152.

20. Gargiulo P, Pétursson T, Magnússon B et al. Assessment of total hip arthroplasty by means of computed tomography 3D models and fracture risk evaluation. Artif Organs 2013; 37: 567-73. doi: 10.1111/aor.12033. Epub 2013 Apr 2.

21. Ewen AM, Stewart S, St Clair Gibson A, et al. Post-operative gait analysis in total hip replacement patients - A review of current literature and meta-analysis. Gait Posture 2012;36:1-6. doi: 10.1016/j.gaitpost.2011.12.024. Epub 2012 Mar 10.

22. Petersen MK, Andersen NT, Mogensen P, et al. Gait analysis after total hip replacement with hip resurfacing implant or Mallory-head Exeter prosthesis: a randomised controlled trial. Int Orthop 2011;35:667-74. doi: 10.1007/s00264010-1040-6. Epub 2010 May 16.
23. Hodt-Billington C, Helbostad JL, Vervaat W, et al. Changes in gait Symmetry, gait velocity and self-reported function followings total hip replacement. J Rehabil Med 2011;43:787-93. doi: 10.2340/16501977-0849.

24. Olsson E, Olsson E, Goldie I, Wykman A. Total hip replacement, A comparison between Cemented (Charnley) and Non-cemented (HP Garches) Fixation by clinical Assessment and objective Gait Analysis. Scandinavian J Rehab Med 1985;18:107-16.

25. Pétursson Th, Gargiulo $\mathrm{P}$, Magnússon $\mathrm{B}$, et al. Bone and muscle assessment in patients undergoing total hip arthroplasty using HU based analysis. Basic Appl Myol 2012;22:147-52.

26. Lindner $\mathrm{T}$, Krüger $\mathrm{C}$, Kasch $\mathrm{C}$, et al. Postoperative Development of Bone Mineral Density and Muscle Strength in the Lower Limb After Cemented and Uncemented Total Hip Replacement. Open Orthop J 2014;8:272-80. Published online Sep 15, 2014. doi: 10.2174/1874325001408010272

27. Gargiulo P, Kern H, Carraro U, et al. Quantitative color three-dimensional computer tomography imaging of human long-term denervated muscle. Neurol Res 2010;32:13-9. doi: $10.1179 / 016164109 X 1253604242417$. 